\title{
Germanica
}

\section{Lars Anderssons Werk: Ausdruck und Möglichkeit einer Transformation der Moderne}

L'oeuvre de Lars Andersson : expression et possibilité d'une transformation du modernisme

Uwe Ebel

\section{(2) OpenEdition}

\section{Journals}

Édition électronique

URL : http://journals.openedition.org/germanica/1498

DOI : $10.4000 /$ germanica. 1498

ISSN : 2107-0784

\section{Éditeur}

Université de Lille

Édition imprimée

Date de publication : 1 juin 1993

Pagination : 143-165

ISSN : 0984-2632

Référence électronique

Uwe Ebel, «Lars Anderssons Werk: Ausdruck und Möglichkeit einer Transformation der Moderne », Germanica [Online], 12 | 1993, Online erschienen am: 06 Juli 2012, abgerufen am 06 Oktober 2020.

URL : http://journals.openedition.org/germanica/1498; DOI : https://doi.org/10.4000/germanica.1498

Ce document a été généré automatiquement le 6 octobre 2020.

(c) Tous droits réservés 


\title{
Lars Anderssons Werk: Ausdruck und Möglichkeit einer Transformation der Moderne
}

\author{
L'œuvre de Lars Andersson : expression et possibilité d'une transformation du \\ modernisme
}

Uwe Ebel

Das Echo, das die Moderne, ihre Kunst, Musik und Literatur, seit sie vor ein und einem halben Säkulum als eigengestaltige Größe erkennbar wurde, hervorrief, blieb die Gegenstimme. Der Tenor solcher Gegenstimme lag in der Ablehnung einer Kompliziertheit, als die die der Moderne eigentümliche Komplexität wahrgenommen wurde. Heute, in einer Phase, in der die Moderne an ihr Ende gekommen und ihre Darstellung auf die Tonlage des respektvollen Abgesangs gestimmt ist, tritt Kritik der Moderne unter anderen Gesetzen und Vorzeichen an. Sie wird, nachdem sie lange Zeit Verurteilung war, Beurteilung, Sichtung. Dort, wo zeitgenössische Moderne attackiert wird, trifft das Verdikt eine Literatur, die sich nicht mehr durchsetzen muß, erwächst es aus der ehemals für die Moderne selbst geltenden Position derer, die sich durchsetzen mußten, denen Anerkennung verweigert wurde. Wenn deshalb Cornelis Vreeswijk in Getinghonung Provençale oder in Ballad om censuren die durch Kritiker und Preisvergabe-Juries verwaltete Zuordnung $\mathrm{zu}$ Kunst oder NichtKunst als Zensur begreift, wendet er sich dagegen, daß in den zuständigen Gremien das schiere Merkmal «unverständlich», «amimetisch», «kompliziert» schon das Konnotat «künstlerisch bedeutend» besitzt. Er steht damit in einer Front mit John Lennon, von dem der Ausspruch stammt: «Avantgarde is just a french word for bullshit»1, und mit Udo Lindenberg, der heute als avantgardistisch ausgegebene Komponisten als «verbogene Avantgardisten» und deren Musik als «alles, was intelligenten Krach macht» ${ }^{2}$, einordnet. Solche Unmutbekundungen wenden sich durchaus nicht ab, um ins Gestrige zurückzukehren. Sie reagieren auf eine plane und platte Fortsetzung der Moderne, deren Aktualität nur noch die des Modischen und Epigonalen ist. Sie widersprechen nicht, sondern korrelieren einer Kritik, die bewußtmacht, daß an der so gescholtenen 
Moderne etwas war, das unhintergehbar ist. Wenn das, was heute das Anstehende ist, sich selbst auf den Begriff der Postmoderne bringt, definiert es sich selbst aus der Figur des Sich-Abhebens und profiliert die Moderne damit als die Strömung, die zumindest für den europäisch-amerikanischen Bereich das Bewußtsein in der letzten größeren Phase am weitesten vorangetrieben hat.

2 Sucht man danach, was an der Moderne unhintergehbar ist, muß man danach fragen, was das Spezifische der Moderne, was das Spezifische ihrer Literatur war. Dabei gelangt man immer wieder auf den Begriff des Experiments. Wie Göran Palm in einer Verteidigung der neuen Einfachheit in fragloser Selbstverständlichkeit von «modernismens experimentella anda» ${ }^{3}$ sprechen konnte, so wird in einer der zahlreichen Verabschiedungen der Moderne, die derzeit erscheinen, der Einwurf gegen sie erhoben, das Experiment sei als solches nicht länger ein Argument ${ }^{4}$. Dem entspricht es, daß in Peter Lutherssons Buch über die Moderne die Belege dort besonders dicht werden, wo Luthersson die Definitionen der modernen Literatur als experimentelle Literatur zusammenstellt ${ }^{5}$. Bei aller Divergenz der Bestimmungen dessen, was die Moderne als eigengewichtige, als eigenständige Strömung ausmacht, besteht offenbar Übereinstimmung darin, daß ihre Kunst, ihre Musik, ihre Literatur experimentell sind, so daß experimentelle Literatur zu einem Synonym für moderne Literatur hat werden können.

3 Die Spezifizierung von Kunst, von Literatur als experimentell ist jedoch indefinit und vage, solange ungeklärt bleibt, wozu das Experimentieren dient, denn als Versuch, als Erprobung kann das Experiment eo ipso nicht Selbstzweck sein. Gewichten wir es deshalb als Symptom, stellt sich die Frage danach, wofür es symptomatisch ist.

Die Geisteshaltung fraglosen Hinnehmens dessen, was allgemein angenommen wird, kennt die Unruhe des Experimentierens nicht. Experimentieren wird man vielmehr dann, wenn Geltendes seine Gültigkeit verliert oder noch nicht Geltendes auf seine eventuelle Gültigkeit hin überprüft werden soll. So treibt denn eine Bewußtseinslage, der die überkommenen Sinnangebote brüchig und fadenscheinig werden, auch eine Literatur des Experiments aus sich hervor. Diese Erklärung ist vorläufig, wohl auch banal und wird erst aussagekräftig, wenn sie den spezifisch literarischen Charakter solchen Experimentierens erklären kann. Es wäre mit anderen Worten zu reflektieren, was es bedeutet, mit und in Literatur zu versuchen und zu erproben. Es bedeutet, daß die Medialität des Mediums, also auch des Mediums Literatur, bewußt wird, daß die Medialität als das Wesen des Mediums absolut gesetzt und das Funktionieren von Verständigung aus der Abbildhaftigkeit auf eine Eigenbildlichkeit verlagert wird. Dieses Bewußtsein wird in der Dichtung im Erbe und Gefolge der Romantik erreicht und dem Vertrauens ver lust inkorporiert, der in der Moderne alle Sinnvorgaben betrifft, so daß auch die Sprache und mit ihr alle Formen des Abbildens und inter subjektiv vermittelbaren Reagierens auf Welt unter diesen Vertrauensverlust fallen. Das Wissen um die Vehikularität, wie es aller Sprachreflexion latent innewohnt, wird in ein Wissen um die unaufhebbare Vermitteltheit dessen weitergeführt, was in Sprache ausgedrückt wird. Freund und Feind der Moderne lagen ihre Argumente um dieses Problem an, und kennzeichnen es damit als das, was es war: das Zentrum.

5 Die Opponenten der Moderne beharren auf der Gegenannahme, daß Sprache Wirklichkeit treffe, und verfügen in der Theorie oder praktizieren im künstlerischen Werk die diversen Ausformungen des Realismus, denen allen ein Vertrauen in die Sagbarkeit von Welt gemeinsam ist. Von Stund an stehen sich realistische und 
experimentelle Kunst antithetisch gegenüber. Da Erkenntnistheorie ein Aspekt einer Ontologie und einer Welthaltung ist, ergab es sich, daß sich die Vertreter der Realismus-Positionen meist feindlich und aggressiv verhalten, ja, wo es machbar war, ihr Anathema in Verbot und Verfolgung vollstreckten: denn wer das Prinzip der Starre vertritt, sieht sich durch Aufbrüche bedroht.

Vor dem Hintergrung des hier Skizzierten wird deutlich, warum sich die massive Kritik, die George Steiner in unseren Tagen gegen die Moderne vorgebracht hat, an deren Sprachauffassung entzündet. Steiners 1989 unter dem Titel Real Présences ${ }^{6}$ vorgelegte Auseinandersetzung gewinnt ihr Gewicht dadurch, daß sie das Zentrum der sich zur Moderne versammelnden Theorien und Theoreme trifft; sie gewinnt Gewicht ferner dadurch, daß Botho Strauß der deutschen Übersetzung von Steiners Arbeit ein zustimmendes und die Abwehr noch weitertreibendes Nachwort beigefügt hat. Die durch Steiner vertretene Kritik an der Moderne wird mithin von einem jener «Erben der Moderne» getragen, von denen Strauß in Paare, Passanten so spricht, daß man ihn selbst zu ihnen zählen muß ${ }^{7}$. Steiner fokussiert den Kollaps des Vertrauens in die Übereinstimmung von Wort und Welt als das Diskrimen der Moderne. Sprache, die noch im rigiden Skeptizismus mit der Welt im Vertrag stehe, «insofern, als... [der Skeptizismus] danach strebte, sich auszudrücken» (127) werde in der Moderne das Vertrauen entzogen, das Vertrauen darauf, Welt sei in ihr sagbar und erzählbar. «Ich bin der Überzeugung», schreibt Steiner, «daß dieser Vertrag zum ersten Mal in irgend fundamentalem und folgenreichem Sinne während der Jahrzehnte zwischen 1870 und 1940 in Kultur und spekulativem Bewußtsein Europas, Mitteleuropas und Rußlands gebrochen wird. Es ist dieser Bruch des Kontraktes zwischen Wort und Welt, der eine der wenigen echten geistigen Revolutionen in der Geschichte des Westens darstellt und durch den sich die Moderne definiert» (127). Das erhält seine bestimmte Wendung, wenn Steiner schließt: «Ich definiere diese Zeit als die des Epilogs» (128). Steiner diskutiert, und darauf bezieht sich solche Kategorisierung, den wenig beachteten «Akt oder Tenor des Vertrauens, das der linguistisch-diskursiven Substanz unserer westlichen hebräischattischen Erfahrung zugrunde liegt, ja sie buchstäblich beglaubigt». Und da heißt es dann: «Oftmals unbeachtet, weil so eklatant resistent gegen Formalisierung, bleibt auch der Kern des Vertrauens innerhalb der Logik selbst, wo "Logik" doch ein vom logosabgeleitetes Konstrukt ist» (123). Das hat bei Steiner, man spürt es schon an der Kategoriensummation, geschichtsphilosophische Konsequenzen. «Bis $\mathrm{zu}$ jener Mutation von Werten, die ich analysieren möchte, "trafen" sich logos und Kosmos» (124), heißt es, und ferner: «Der Vertrag zwischen Wort und Gegenstand, die Voraussetzung, daß das Sein in einem praktikablen Maße "sagbar" ist und daß das Rohmaterial der Existenzialität sein Analogon in der Struktur von Erzählung hat - wir erzählen Leben, wir erzählen es uns selbst -, hat immer wieder Ausdruck gefunden» (124).

7 Steiner sucht aus diesem Dilemma auszubrechen, indem er die Immanenz des Denkens aufzuheben - in seinem Sinn weitergedacht müßte man sagen zu überwinden - sucht, wobei sie ihm zum Teufelskreis wird, der folglich in Acht und Bann zu tun sei. Immanenz wird durch eine von Steiner vorgenommene Vektorverschiebung in Richtung auf Begrenzung, auf Einengung dimensioniert. Entsprechend stellt sich ein Negativwert ein, der auf einen Schaden verweist, den es aufzuheben gelte. Der 
Positivwert ist der der Grenzüberschreitung, der auf dem Weg des Trans-zendierens zu einer Transzendenz hinführt, die in sprunghafter Gedankenführung und als «Schöpfer» spezifiziert substantialisiert wird. Nicht von ungefähr lehnt Steiner zu Beginn seiner Arbeit eine Sprachanalyse ab, die darauf zielt, sprachlich bedingte Gedankenfehler aufzuspüren. Hier ereignet sich - unausgeführt und verborgen - die restitutio jenes in der Moderne aufgegebenen Vertrauens in die Übereinstimmung von Wort und Welt. Was dabei allerdings als restitutio in integrum angeboten wird, ist lediglich die Wiedereinsetzung in den alten Stand. Das rechtfertigt sich bei Steiner daraus, daß der verhandelte Sachverhalt als Vertragsbruch ausgelegt wird. Erörtert und bedenkt man den Sachverhalt aber als Einsicht in die Vermitteltheit allen Sprechens von Welt, in die Medialität und Vehikularität des Sagens und Sprechens, entpuppt sich die Einsetzung in den vorigen Stand als Rückkehr in die Illusion.

Der Steiner sehen Kritik wächst eine andere Wertigkeit, auch eine andere Wirkmöglichkeit $\mathrm{zu}$, als die eines Beitrags zu einer innerfachlichen Diskussion der Moderne. Dazu verhilft ihr schon die verlegerische Präsentation, die über die eines Fachbuchs hinausgeht, und die Beigabe einer radikalisierenden Zustimmung durch einen so bekannten Autor wie Botho Strauß. Sie verdient deshalb verstärkte Beachtung. Da sie sich als Zeitdiagnose mit Therapievorschlägen versteht, da sie in ihren Schlüssen antidemokratisch, antirational, ja biologistisch ist, stellt sich die Frage, ob die Konsequenzen aus der Analyse zwingend abgeleitet sind, oder ob das, was hier als Konsequenz angeboten wird, nicht doch vorgängig war und die Analyse steuerte.

Im Zweifel, also auch im «Zweifel an der Sprache» (Erich Fried), ist eine Ambiguität der Sicht gewahrt. Aufgegeben ist zunächst allein die Sicherheit der Annahme, daß Sprache Wirklichkeit erreichen könne. Die Offenheit, die in der Frage, ob sie es könne, noch gewahrt bleibt, läßt die Metamorphose dieser Frage zur Frage danach zu, wie Sprache es dennoch vermag, von Welt zu sprechen. Wenn heute eine Literatur entsteht, die die Erkenntnisse und Einsichten der Moderne nicht aufgibt, sondern weitertreibt, indem sie die Kritik an der Moderne in ihr eigenes Bewußtsein aufnimmt, dann wägt sie die Prämissen des Realismus und die der experimentellen Literatur gegeneinander $\mathrm{ab}$ und sucht nach einem Weg, den in beiden vorhandenen Wahrheitsgehalt zu verbinden. Ihr wird der Zweifel an der Sprache seinerseits zum Problem, ohne deshalb die Abwehr eines «a priori berättande», die Gunnar Björling ${ }^{8}$ formuliert hat, einfach zu umgehen, ohne dem Anspruch «att det ej ges en given form i sig» (178) zu widersprechen, in dem derselbe Gunnar Björling «det modemas väsen» (178) sah. Es wäre also eine Literatur nicht mehr der Modernen, sondern der - um die oben zitierte Kategorisierung von Botho Strauß aufzugreifen - «Erben der Moderne».

Daß die Folgerungen, die Steiner aus seiner Kritik der Moderne zieht, nicht zwingend sind, zeigt, daß seine Problematisierung bestimmter Denkansätze der Moderne in Parallele, seine Lösungsvorschläge angesichts gewisser Defizite solcher Denkansätze hingegen in Kontrast zu den Arbeiten eines der zweifellos bedeutendsten schwedischen Autoren unserer Tage stehen. Die Rede ist von Lars Andersson, der das von Steiner Erarbeitete als Dilemma der Moderne ebenfalls erkannt, es aber nicht gegen die Moderne gewendet, sondern als Korrektiv an ihr erarbeitet hat, womit sein Werk, Essayistik wie Epik, zur Weiterführung der Moderne wird, die zugleich deren 
Überwindung ist. In seinem Werk ist das Experiment in eine Form überführt, die es, um die oben zitierte Kritik aufzugreifen, durchaus wieder zum Argument werden läßt.

11 In seinem Aufsatz von 1987 über «Das moralische Gen»'setzt sich Lars Andersson mit der Annahme der Biosoziologie auseinander, Denken und sittliches Empfinden des Menschen seien Teilbereiche der Anpassung des Menschen an seine Umwelt, mithin biologisch bedingt und bestimmt. Wenn Andersson diese Theorie in die Enge treibt, dann nicht im inner fachlichen Diskurs, sondern der Konsequenzen wegen, die sie für Erkenntnistheorie und Ontologie hat. Sie hat, wie zu zeigen sein wird, Konsequenzen für die Theorie der Sprache und auf diesem Weg für die Praxis der Dichtung. Andersson geht auf die Zäsur ein, die der Darwinismus für Erkenntnistheorie und Ontologie bedeute, insofern mit dem Darwinismus die theologisch argumentierende Theorie einer angemessenen Weltaneignung durch und im Denken ins Wanken gekommen sei. Die Zielrichtung seiner Auseinandersetzung mit solchen Theorien ist der Nachweis dessen, daß Welt menschlichem Denken zugänglich ist, daß die Rationalität des Menschen der Rationalität der Welt korrespondiert. Aus diesem Impetus ergibt sich die Emphase, mit der Andersson darauf verweist, die Mathematik habe bewiesen, daß Erkenntnis der Welt durch «rena logiska abstraktioner och tankelekar utan varje likhet med känd verklighet» (59) - Wirklichkeit im Sinn von empirisch erfahrener Wirklichkeit - zu gewinnen sei. Er konstatiert solche Denkbar keit nicht nur, sondern zieht Folgerungen, in denen das eigentliche Anliegen seiner Auseinandersetzung mit der Biosoziologie zutage tritt: «Här tycks finnas en överensstämmelse mellan tanke och värld som är oförklarlig. [...] Då måste man [...] i bilden av våra villkor införa nåt som är och förblir oförenligt med varje darwinsk övertygelse, sociobiologisk eller inte: finalitet, avsikt, och alltså i nån mening Skapelse» (59). Wenn Andersson den Begriff der Schöpfung wieder aufgreift, folgert er Metaphysisches nicht allein im «übertragenen» Sinn einer Metapher oder gar Exmetapher. Er bricht die Diskussion in diesem Essay damit jedoch einstweilen ab, nicht ohne eine Richtung des Denkens anzugeben: «Och då är steget snubblande möjligt in i även en etik ontologi, en som inte "sitter i generna" men ändå hör oupplösligt ihop med människans art. Men det steget för ut ur den här diskussionen» (59).

Das in diesem Essay sich äußernde Problembewußtsein ist dem verwandt, das sich in Steiners Buch zeigt: hier wie dort geht es um Logik, um Übereinstimmung von Gedanke und Welt; hier wie dort wird thematisch, daß diese Übereinstimmung durch theoretische Ansätze des 19. Jahrhunderts in Zweifel gezogen worden sind; hier wie dort wird dieser Zweifel kritisch bedacht; hier wie dort wird wieder ein Schöpfer bzw. eine Schöpfung in das Denken eingeführt. Das aber ist eher eine Ähnlichkeit der Absicht, deren Duplizität darauf verweisen mag, daß sie ein überindividuelles Bedürfnis spiegelt. Die weitere Argumentation divergiert jedoch stark. Bei Steiner führte Logik zum Begriff des Logos, Logos zu dem des Worts, Wort zu dem der Sprache. Andersson spricht nicht von einer Ubereinstimmung von Wort und Welt, sondern von einer Ubereinstimmung von "Gedanke und Welt». Es geht also um das vorrangige Problem der Denkbarkeit von Welt. Dieses Problem ist in Anderssons Ansatz prinzipiell gelöst, nicht aber das Problem der Sagbarkeit von Welt - wenn man Sagbarkeit in der Form des Sprechens mit Mitteln der Sprache im engeren Sinn versteht. Die Lösung dieses Problems ist in der Lösung des unmittelbar verhandelten Problems soweit vorgegeben, als Andersson eine Form des Abbildens von Welt diskutiert, nämlich die der Mathematik. Die mathematische Formel leistet Darstellung für einen Teilbereich der Welt, indem sie die Rationalität ihres Gegenstands formuliert. Sie ist - platonisch 
gedacht - die Idee des Phänomens. Damit bildet sie im strengen Sinn jedoch gerade nicht ab, sondern bringt auf den Begriff, ist selbst, was sie ausdrückt. Im Konzept des Logos, der am Anfang war, des Worts, das erschaffende Kraft besaß oder adamitisch das Wesen des Benannten erfaßte, nimmt Sprache dieselbe Stelle ein wie die Mathematik in ihrem Bereich: sie bringt die Rationalität, die Logik zum Ausdruck, die objektiv in der Welt vorhanden ist, sie ist, was sie benennt, im Seinsstatus der Idee. Hier liegt die Falle, in die Steiner geraten ist, weil er die verschiedenen Bedeutungen, die das Wort logos unabhängig von seiner ursprünglichen Semantik entwickelt hat, ineinanderschiebt. Logik ist nicht durch das gesprochene Wort verbürgt, wie könnte man sonst mit Worten lügen; die Sprache der Mathematik, regelgerecht gebraucht, schließt Lüge aus.

Geht man von den in diesem Essay Anderssons gewonnenen Belegen für die Denkbarkeit der Welt aus, ergibt sich aus solcher Annahme die Sagbarkeit von Welt als Postulat. Damit ist Sprache ins Zentrum gerückt, ohne daß der schlichten Wiedereinsetzung der Sprache in ihren alten, vor-modernen Status damit ein Weg gebahnt wäre. Um Anderssons Ansicht über die Sprache, über deren kommunikative Leistungsfähigkeit zu ermitteln, muß man zu anderen Texten greifen. Andersson problematisiert Sprache immer wieder, und zwar in zwei Richtungen, wenn er einmal danach fragt, wie sich Mensch und Mensch zur communio kommunizieren, und zum anderen, wie mit Hilfe von Sprache Äußerungen über Außersprachliches zustande kommen können. Letzteres meint den mimetischen Charakter des Sprechens.

In seinem Aufsatz «Till kritiken av kritiken» ${ }^{10}$ von 1978 schreibt Andersson: «Av allt att döma går vi in i en ny romantik, och jag vill gärna stå i startgroparna och säga: Jag tror att konsten i sig är revolutionär!» (152). Der Zusammenhang, in dem diese Äußerung bei Andersson steht, ist der einer Diskussion darüber, wie im literarischen Kunstwerk auf Wirklichkeit so verwiesen werden kann, daß sie darin erkannt werde. Damit ist das Problem der Darstellung von Wirklichkeit wieder bewußt und, da als Problem anerkannt, prinzipiell auch als lösbar gesetzt. Indem Andersson das Problem stellt, gibt er das Wissen um die Vermitteltheit der Abbildung von Welt im Medium der Kunst hier des Romans - nicht auf, er übernimmt eine in der Moderne gewonnene Position, ohne sie dogmatisch zu verabsolutieren. Er schreibt, und der Zusammenhang ergibt sich aus der Problematik der Darstellung der für die siebziger Jahre spezifischen Angst: «Litteraturen kan naturligtvis spela någon liten roll som ideologisk röst. Men om den ska få nån verklig betydelse tror jag att den framför allt måste formalisera detta rädda 70-tal, tematisera, strukturera, mytologisera det. Låta rädslan ta en gestalt, transformeras genom en myt, så att den blir möjlig att överblicka, genomskåda, överskrida» (151). Andersson stellt das Konzept des Romans auf, «som gör läsaren mytologiskt medskapande och överskridande» und sieht darin «ett experiment i att göra denna verkliga värld tänkbar - och därmed överskridbar, möjlig att förvandla» (151sq).

Das literarische Verfahren ist das des Experiments, nun jedoch eines Experiments, das mit der Zielvorstellung angegangen wird, auf Welt zu verweisen. Man kann sich den Schlußfolgerungen durch folgende Operationen nähen, die zunächst Gedankenspiele sind, aber - falls konsistent - auf Reales verweisen müssen. Mathematik und Literatur bilden Welt ab. Mit der Moderne verlassen Mathematik und Literatur ihre Bestimmung als Weltabbild. Sie entwickeln sich zu Formen weiter, die auf keine Realität mehr zu verweisen scheinen. Man könnte den literaturbezogenen Terminus auch auf die Mathematik anwenden; beide werden autoreferentiell. Die mathematischen 
«Gedankenspiele» erweisen sich im nachhinein dennoch als weltabbildend. Das hat Bedeutung für die Weltauffassung: sie ändert sich aufgrund der mathematischen Operationen. Literatur scheint sich auch von der gegebenen Wirklichkeit entfernt $\mathrm{zu}$ haben. Sie könnte dabei auf dem Weg zu einer Weltabbildung sein, die «verborgene» Seiten der Welt darstellbar werden läßt. Euklidische Mathematik erfaßt abbildend lebens weltliche Empirie. Euklidische Mathematik verhält sich zur lebensweltlichen Empirie wie nicht-euklidische Mathematik und Mathematik der irrationalen Zahlen zur lebensweltlich nicht erfahrbaren Welt. Euklidische Mathematik verhält sich zur nichteuklidischen Mathematik wie Realismus zu experimenteller Ausdrucksform. Umgekehrt gälte: Realismus ist Ausdruck einer begrenzten Weltsicht des Alltagsverstandes im beschränkenden Sinn. Realismus hält sich beim Auswendigen auf, beim Schein. Die Rationalität, die Logik (Steiner) der Welt erfaßt der Realismus gerade nicht. Im Thematisieren, also begrifflich klärenden Erkennen, im Strukturieren, im Formalisieren, schließlich Mythologisieren wird Realität einem Abstraktionsgrad zugeführt, der sie erkennbar macht, erkennbar in einem höheren Maß als im Typisieren etwa des sozialistischen Realismus. Solches Mythologisieren verfährt induktiv, nicht deduktiv und nimmt darin die Kritik am Mythos in sich auf, ist nicht selbst mythisch.

Die frühe Moderne, so etwa Baudelaire, so etwa Jens Peter Jacobsen, befaßte sich gründlich mit Edgar Allan Poe. Poes Essay The Philosophy of Composition ist insoweit als Programmschrift der Moderne zu betrachten, als hier der Weg des Abbildens von Welt verlassen und eine Literatur vorgestellt wird, die nicht darauf abzielt, die Realität auf ihre Idee zu beziehen, sondern eine Idee zum Phänomen zu gestalten. Poe kehrt die adamitische Sprachauffassung um: nicht im Berufen wird Welt beim Namen genannt und so auf das Wort bezogen, das sie erschuf; im sprachlichen Erschaffen einer nur in Sprache existenten Realität wird eine Idee in eine angemessene Erscheinung umgesetzt. In Anderssons Diskussion wird nun wieder zu einer Phänomenalität die Idee gesucht, die in einer Weise dargestellt werden soll, daß sie in der Phänomenalität solcher Darstellung wiedererkennbar wird. Hier schließt sich ein Kreis, besser, hier wird die Entwicklung um eine Spiralführung weiter getrieben.

Lars Anderssons Romane führen ein Geschehen vor, das als real fingiert und referierbar ist. Dieses Geschehen ist jedoch in seiner Struktur experimentell ausgerichtet, so daß in seiner Kompliziertheit eine Komplexität entsteht, die dem realistischen Erzählen fremd ist. Was in diesen Romanen als kompliziert, als verständniserschwerend erlebt wird, ergibt sich nicht aus einer verwickelten Handlungsführung mit Nebenhandlungen, Zeitsprüngen oder ähnlichem, wenngleich die Romane mit Zeitsprüngen arbeiten, sondern aus einer Sinnfülle, die mit Mitteln innerliterarischer Verfahren hergestellt wird, die das Realistische bedrohen und latent wieder aufheben. Die Sagbarkeit von Welt oszilliert zwischen der Erzählbarkeit von Vorgängen, die als real fingiert sind, und der Konstruktion von Zusammenhängen, die die einzelnen Momente aufeinander beziehen. Dieses In-Bezug-Setzen geschieht mit einer Rigidität, die jeglicher Weltbetrachtung widerspricht, wie sie die Verfahrensweise realistischer Literatur fundiert, und wird seinerseits durch ein Zusammenhänge suchendes Ich verbürgt. Es entsteht der Typ eines monologischen Romans, der sich dem dialogischen Roman, den Michail Bachtin als einen der Prototypen realistischer Literatur ermittelt hat, diametral 
gegenüberstellt. Anderssons Roman experimentiert im angestrengt aufmerksamen Lauschen nach konzertierendem Widerhall, den das treffende Wort dazu befähigt, in allem Mitbetroffenen ein Echo zu finden. Er experimentiert im Kombinieren des Eigenen und des Zitats zur sich ergänzenden, sich absprechenden Rede. Auch darin ist das Verfahren antirealistisch, wenn man das von Bachtin etwa bei Dostojewski aufgefundene Stimmengewirr ununterbrochen abgerufener und aufeinanderprallender fremder Rede als unverzichtbares Moment realistischer Literatur akzeptiert. Fernzuhalten ist dabei mit allem Nachdruck die Anmaßung, das Abrufen divergierender und oft nicht zur Harmonie zu bringender Rede, also das, was dann von Bachtins Nachrednern immer wieder als das Dialogische erarbeitet wird, sei im Gegensatz zu anderen Verfahren demokratisch. Solche Etikettierung ist nicht verdeutlichend, sondern veredelnd.

Anderssons Romane - sein Verfahren sei an Löv till läkedom exemplifiziert ${ }^{11}$ - kreisen um Kernbegriffe, um Themen, die sich ihrerseits um einen gemeinsamen Mittelpunkt konzentrieren. Sie werden in Löv till läkedom in Gesprächen diskutiert und in literarischen wie halbliterarischen Texten behandelt, die Figuren des Romans selbst schreiben oder zu schreiben beabsichtigen. Der Roman stellt mehrere Zweiergespräche in den Mittelpunkt seines ersten Teils. An ihnen ist jeweils der Schriftsteller Per Ivar Ödman beteiligt, der die Thematik bestimmt und, wenn er geduldig und interessiert zuhört, Reaktionen auf die ihn beunruhigenden Probleme provoziert. In diesen Gesprächen wird die Bedeutung, die die Genforschung für den Begriff vom Menschen hat, erörtert, es wird eine Theorie der Geschichte, besser eine Theorie der Rationalität oder Logik der Geschichte erarbeitet; es wird über das Erzählen diskutiert und eine Theorie des Erzählens entwickelt. In diese Themen eingeschlossen sind die der Schöpfung und des schöpferischen Akts im religiösen und im säkularen Sinn, das Thema der Identität und des Ich, der Kommunikation und der Utopie, der Sinnleere wie der Sinnfülle von Geschichte, der religiös verbürgten Hoffnung auf einen glücklichen Ausgang der mißglückten Geschichte. Ihre Erörterung wird in Notaten und Entwürfen weitergeführt, als deren Verfasser geschehensimmanent Ödman zu denken ist ${ }^{12}$, deren Charakter als Notizen Ödmans aber in der Präsentation des Textes zurücktritt ${ }^{13}$. Indem der Text diesen Charakter zurücktreten läßt, relativieren sich diese Notate nicht zur Meinung einer der Figuren, wie überhaupt die in den Diskussionen geäußerten Ansichten nicht denkbare oder historisch virulente Positionen repräsentieren, sondern sich als Teilargumente in eine durch den Roman in toto geführte Diskussion fügen.

Das gilt auch für die Wahl und Zuordnung der Themen selbst. Sie sind so gewählt, daß sich ihre Erörterung einem gemeinsamen Diskussionsziel annähert, daß der Roman eine in ihm dargestellte Beunruhigung nach allen ihren Seiten hin abhorcht. Durch dieses Verfahren wird das in den Gesprächen wie in den essayartigen Textabschnitten Erörterte isoliert und konsistent zugleich. Vermitteln sich die Themen auch einerseits als isoliert, als verworren, so geben sie sich andererseits als geordnet zu erkennen, geordnet nämlich nach dem Modell des Spektrums, der Aufgliederung in Facetten. So erhalten sie eine Konsistenz, die bis zur Systematik weitergedacht werden kann. Die Anordnung aber als Facetten läßt das Systematische als Ergebnis erscheinen, präsentiert es als Reaktion auf ein Verworrenes, das als verworren im Bewußtsein bleibt. Weil das in Löv till läkedom Diskutierte sich aber als systematisierbare Teilbereiche einer geschlossenen Theorie darstellt, kann es auf einen gemeinsamen 
Nenner gebracht und zu dem im epischen Geschehen Gestalteten in Bezug gesetzt werden.

Das Ödman beunruhigende Problem formuliert sich als das Problematische der Genforschung, die den Begriff des Menschen fundamental verändert habe. Der Mensch entwickelt den Menschen nach Physis und Psyche den Ausgeburten seiner Phantasie zum Bilde. Wie in Massenschlacht und Vernichtungslagern die Willigkeit des Menschen zur Ausrottung des Menschen bekundet wird, die in der Entwicklung von Massenvernichtungswaffen total werden kann, so bekundet sich in der Genmanipulation die Willigkeit des Menschen, den Begriff vom Menschen zu vernichten. Die darin sich entäußernde Logik der Geschichte wird nun auf ein in ihr anwesendes spezielles Vermögen des Menschen hin befragt. Der Roman gelangt an dieses Vermögen über den Begriff der «Spaltung», der nach Aufspaltung und Abspaltung, dann nach Sonderung, Absonderung und Aussonderung weiterentwickelt wird. Zunächst spaltet sich ab, wer sich absondert. Wer sich in einer Besonderheit erkennt, identifiziert sich, setzt eine Identität. Sie impliziert, daß ein anderes erkannt werden kann, dem sich ein Ich gegenüberstellt, das es objektiviert. Um ein anderes objektivieren zu können, muß ein Bild von ihm erstellt werden, muß es bezeichnet werden können. Die älteste Form einer bildhaften Verständigung über Welt ist der Tanz, «nånting annat än de genetiskt inpräglade rörelsemönstren» (174); ihm folgt in der Höhlenmalerei die bildhafte Darstellung gejagter und zu jagender Tiere. Solcher Identifizierung des anderen geht die Körperbemalung als Versuch, sich eine Identität zu schaffen, voraus. Indem ein Ich etwas anderes setzt, indem ein Ich sich selbst setzt, grenzt der Mensch sich aus dem umgreifenden Zusammenhang der Lebewesen aus. Er setzt zugleich Differenzen unter den Menschen. In solch früher Phase des Prinzips der Individuation ist noch keine radikale Abspaltung des Menschen aus dem umgreifenden Lebenszusammenhang gegeben. Der Mensch beschwört in der Höhlenmalerei die Tiere, indem er sie malt, und ist mit ihnen noch in einer übergeordneten Gemeinschaft verbunden. Erst als der Mensch seßhaft wird, spaltet er sich ab, «stößt er sich ab» von dem, was ihn vorher noch in sich barg, und tritt in die Geschichte ein.

Der Roman setzt in Bezug, was Beziehungen zuläßt, er tut das im Sinn des oben benannten Verfahrens des Widerhalls. Das ermuntert, ihn beständig weiterzudenken, Beziehungen herzustellen, auch wenn sie nicht immer unmittelbar im Roman selbst formuliert sind. Immerhin heißt es vom Höhlenmaler expressis verbis, daß die Tiere, die er gemalt habe, «byte, lärare, gudavareilser» (176) gewesen seien. Von Linné wird gesagt, er habe die Tierwelt von außen geordnet, in seiner Darstellung überlagere die Art das Wesen (53). Die Fähigkeit des Menschen, abzubilden, darzustellen, das mimetische Vermögen also, ist, so muß man schließen, vor jener "passage till den historiska tiden» (79) noch nicht von dem Verhängnis des Spaltens, des Auf spaltens und Abspaltens ereilt. Nach jenem historischen Einschnitt, der u.a. zu Linnes Tier- und Pflanzenlehre geführt hat, ist die Darstellung nur noch Gegenüberstellung, Absonderung: Mensch und Nicht-Mensch stehen sich gegenüber, begreifen dieses Gegenüber als hierarchisch begründet, machen den Aspekt «Beute» exklusiv. Daß Menschen eine Identität suchen, hat dieselbe Doppelheit in der Einheit; nach jener Zäsur wird die Setzung von Individualität gebunden an die Setzung von Gruppen, die sich ihrerseits hierarchisch ordnen und sich gegenseitig das Lebensrecht absprechen. In den Entwürfen zu Artedi/Majorana wird der Tod im Wasser als Rückkehr zu den Anfängen ins Bild gesetzt: Artedi, der in der Epoche Linnés und in dessen Verfahrensweise die Fische abbildend ordnet, kehrt in die Welt des Wassers, aus dem 
das Leben kam, zurück, entfernt sich aus Zeit und Raum, ruht in der Haltung des Embryos. Majorana, der das Atom gespalten hat, eine Spaltung, der der Text an anderer Stelle die Spaltung des Begriffs vom Menschen zuordnet, kehrt ebenfalls in das Wasser zurück, sucht subjektiv aufzuheben, was er objektiv nicht rückgängig machen kann.

Wie Artedi und Majorana im Wasser ihren Tod finden, sich der Welt und der Geschichte entziehen, so - und dieses Bezüge-Setzen im beziehenden Adverb ist wohl exegetisch gerechtfertigt - so also entzieht sich Gunnar Elofsson für die Zeit eines Filmfinales, indem er nackt im Wasser verschwindet, der Welt dieses Films, dem Blick der Kamera. Wenn der Fisch für eine frühe Existenzform des Lebens steht - wenn er wohl auch für eines der Wesen steht, die vor jener «Passage in die geschichtliche Zeit» «Beute, Lehrer, Götter» gewesen sein mögen - dann erhellt von hier aus Gunnars Wunsch, zu fischen, der ausdrücklich abgetrennt wird von dem Wunsch, einen Fisch an die Angel zu bekommen.

Ödman war in die ländliche Seßhaftigkeit Gunnars und Sav-reins eingedrungen mit seinen Problemen - einer zerbrochenen Liebesbeziehung, der Beunruhigung über die Möglichkeiten der Biologie, nicht wie die Atomphysik allein die physische Existenz des Menschen, sondern jetzt zusätzlich den Begriff des Menschen zu zerstören. Seine Beunruhigung läßt ihn nach Lösungen suchen, vor Romanbeginn in einer geschichtsverbürgenden Transzendenz, wie sie als Vorsehung in christlicher Religion und neuerer Theologie verhandelt wird, im Roman selbst in theologischen Gesprächen mit Savrein und in Gesprächen mit Gunnar über die Geschichte. Sein Besuch auf dem Land endet damit, daß er sein Problem in einen literarischen Text umsetzt. Optische Eindrücke das Beobachten abgetauten Wassers, das sich fließend teilt und Diskussionsreminiszenzen - Genesis, theologisch und historiologisch bedachte Entwicklung der Geschichte des Menschen - vereinen sich darin zu einem Mythos, der als Antwort auf die Ödman beunruhigenden Fragen gelesen werden kann. Ödman hat eine Aufgabe gelöst und reist ab. Der Aufenthalt bei Gunnar und Savrein hat ihn durch ein Ausstellungsplakat mit der Welt des Judentums in Kontakt gebracht (28). Wohl unmittelbar nach seiner Rückkehr nach Uppsala reist er nach Israel. Hier findet er zu Antworten auf seine Beunruhigung, die sich ihm zu einer Weltdeutung und betrachtung fügen und sich zu dem entwickeln, was der Roman in seiner Gesamtheit an Lösungsversuchen anbietet. Daß der Roman die von Ödman gefundenen Antworten teilt, äußert sich zunächst in seinem Titel. Dieser Titel zitiert eine Wendung aus dem 47. Kapitel des Buchs Hesekiel, das zusätzlich im Roman, ohne die zum Titel erhobene Wendung dort zu übernehmen, als eine Art Hoffnungsmythos referiert wird (206). Das derart berufene Heilmittel wird damit einer Schrift entnommen, in der sich eine Gruppe von Menschen eine Religion geschaffen hat, die imstande war, noch die faktisehe Widerlegung alier in dieser Religion angenommenen göttlichen Versprechen und Verheißungen $\mathrm{zu}$ integrieren, eine Religion, die die Zerstörung der gruppenspezifischen Hoffnungen so $\mathrm{zu}$ reflektieren ermöglichte, daß solche Hoffnungen zu einem Postulat der Gruppenexistenz umgedacht werden konnten. Mythisches im traditionellen Sinn wird zur Strukturvorgabe für einen neu zu formulierenden Mythos der Hoffnung, der die Verzweiflung nicht auf billige Weise überspielt. Eine die Absurdität der Geschichte voll entfaltende Theorie, ein die Negativität des Geschichtsverlaufs unverbrämt ausdiskutierender Roman greift auf ein Heilmittel zurück, greift auf ein Heilmittel vor, dessen heilende Kraft nur noch ein Postulat ist, das, wenn man es seiner religiösen Fundierung entrückt, immer noch ein Postulat der Humanität bleibt. Solcher Rückgriff auf das Alte Testament wiederholt sich 
in der Bezeichnung der Kapitel, deren jedes mit einem Buchstaben der hebräischen Urfassung folgender Wendung aus dem Buch Jesaja versehen ist: «Mit Freuden werdet ihr Wasser schöpfen aus den Quellen des Heils» (Jesaja 12, 3) ${ }^{14}$. Die im Alten Testament festgehaltenen Geschichtshoffnungen strukturieren, prädisponieren mithin das gesamte Geschehen des Romans, insofern der Roman seine Inhalte unter Rückgriff wie unter Vorgriff auf Verheißungen ordnet, die der Roman bereits im Titel abrief und dann auf diese Weise in seiner Kapitelfügung expliziert, auffächert.

Was für den Zusammenhang unserer Diskussion hier wesentlich war, war die Verbindung von Realismus und Experiment: der Roman konstruiert sein Geschehen markant und deutlich, und er erzählt eine als real fingierte Geschichte. Er löst Probleme in einem Erzählverfahren, das explizit auf mythische Erzählmuster und auf bereits fixierte Mythen zurückgreift. Das entspricht einer Theorie des Erzählens, die der Roman selbst diskutiert, wenngleich auf eine nur schwer nachvollziehbare Weise. Gunnar Elofsson erläutert Ödman, was er unter Erzählen versteht, Ödman parallelisiert solchermaßen theoretisch erfaßtes Erzählen seinerseits mit der Sprache im allgemeinen und gibt der Erzähltheorie dadurch einen umfassenderen Zusammenhang. Diese Erzähltheorie wird als eine Theorie der Dichtung formuliert, und ihr wird das Dichten zu einem Mythologisieren, an anderer Stelle des Romans auch $\mathrm{zu}$ einem Formexperiment, womit wir unmittelbar bei unserem Thema wären.

Die Theorie des Erzählens kreist um die Pole Ich, auch als Identität begrifflich gefaßt, und Eindrücke von Welt. Der Normalzustand des Menschen ist der, sich solchen Eindrücken auszuliefern. Da sie ihrerseits durch utspriddhet (78) bestimmt sind, wird das Ich, das sich ihnen ausliefert, ebenfalls konfus, es wird orientierungslos. Als Ich konstituiert es sich in einer Erzählung, und das erzählende Ich erschließt sich Welt als Zusammenhang. An dieser Stelle der Überlegungen greift Gunnar Elofsson auf Sprachtheorie zurück. Die benannten Momente werden in Sprache zusammengefügt, Sprache wird zum Ordnungsfaktor. Da aber Sprache eine problematische Lösung des in der Erzählabsicht und -aufgäbe Gegebenen ist, muß eine angemessene Sprache gefunden werden. Hier werden verschiedene Konzepte aufgegriffen und produktiv gemacht: das Konzept des Mythisierens, das Konzept des Ordnens nach programmierten Vorgaben, im Bild von der Clusteranalyse angesprochen, und das Konzept, das in der jüdischen Theologie und Philosophie mit den Erzählungen verbunden ist, die eine «gleichsam als ob» - Struktur unterlegen und in der Wendung kevejachol diesen Vorbehalt in die Darstellung hineinnehmen, so das Ergebnis als Lösung präsentierend, es aber auch als nicht endgültig und gleichnishaft zu erkennen gebend. Der Text benennt das Verfahren des in der Geste des Kevejachol-Sagens als Remythologisieren einer Frage in der Antwort auf sie. Sprache, Sagen von Welt wäre Ergebnis einer Suche, nicht Benutzen überkommener, zur Konvention gewordener Sageweisen. Sieht man im Bild von der Clusteranalyse mehr als eine mehr oder weniger beliebige Metapher, dann verweist es auf ein Programm, das erst immer zu entwickeln wäre, dem die Formulierung eines Zusammenhangs übergeben würde. Der Text spricht von den Benennungen der Dinge im Wort, sie sind ein Teilvorgang des Sagens, erst ihre Kombination ergibt die zusammenhängende Darstellung. Sprache ist, so heißt es an anderer Stelle des Romans, Ergebnis und Promotor des Vorgangs, der zur Geschichte führt. Sie ermöglicht es, das Ferne, das nicht unmittelbar Vorhandene und Umgebende 
$\mathrm{zu}$ benennen. Sie nimmt den Menschen als das sprechende Wesen aus der selbstverständlichen Eingebundenheit heraus. Verständigung untereinander nimmt andere Dimensionen an, wird Teil der Konventionen, die als Sitte und Gesetz den geschichtlichen Menschen auszeichnen, der die Homöostase der vorgeschichtlichen Lebenszusammenhänge verliert (45). Der - im Sinn des Romans - Verlust einer vorund außersprachlichen, einer noch nicht auf Hilfen angewiesenen Verständigung von Mensch und Mensch geht einher mit der Entwicklung mimetischer Fähigkeiten und Absichten, wie oben referiert.

Wenn von Ödman gesagt wird, daß ihm, dem die Fähigkeit, Komplementärfarben zu sehen, abgehe, die Fähigkeit fehle, Prosa zu schreiben (27), erinnert das an eine Formulierung, mit der Broder Christiansen, eingebunden in das Literaturverständnis der durch die Moderne geprägten Poetik, beschreibt, was Literatur von Wirklichkeit unterscheide ${ }^{15}$. Christiansen, der den Eigengebildecharakter des im literarischen Werk fingierten Geschehenszusammenhangs diskutiert und sich um die Bestimmung dessen bemüht, was Welt von Literatur trennt, sieht das Herstellen von Komplementarität als wesentliches Moment literarischen Sprechens. Das bei Christiansen Erkannte hat in der politisch motivierten Literaturkritik unserer Zeit unter anderer Auswertung nachgewirkt. Christian Enzensberger formulierte sein Verdikt gegen jegliche Literatur unter dem Hinweis darauf, daß sie durch «existentielle Bedeutungsvernetzung» ${ }^{16}$ bestimmt sei und deshalb immer deute und verfälsche. Wenn etwa Franz Koppe Chr. Enzensbergers Auswertung solcher Eigenart des Literarischen die «Perseverenz des Werkcharakters der Literatur $\aleph^{17}$ als den Positivwert von Dichtung entgegenhält, dann ist er nahe bei dem Konzept, das auch in Lars Anderssons Arbeiten und in der von ihm aus formulierten Theorie erkennbar ist, wenngleich das Ergebnis im einzelnen anders ausfällt.

Die Schlüsse, die Christian Enzensberger zieht, wurden in der Moderne unter anderer Gewichtung als das Wesentliche der Sprache und des künstlerischen Gestaltens erkannt. Was bei ihm als Defizit erarbeitet wird, ist die Medialität allen künstlerischen Abbildens von Welt, es ist streng genommen die Medialität des Sprechens. Bei Andersson wird solches Defizit theoretisch und praktisch behoben: theoretisch im Konzept der mythologisierenden Literatur, die auf die Strukturen des Wirklichen, dessen, was ist, aufmerksam geworden ist; praktisch im Konzept der im oben beschriebenen Sinn zur Clusteranalyse greifenden und auffordernden Erzählweise. Die Frage nach Zusammenhängen, die in die Frage nach Zusammenhang hinübergleitet, ist die alle Kunst fundierende Frage, insofern Kunst substantiell Gestalt ist. Darin korreliert Kunst der Sprache, die als Summe von Kategorisie-rungen das Disparate, die disiecta membra zur coniunctio bringt.

Anders aber als in der Moderne, anders erst recht als in deren negativer Umformulierung bei Chr. Enzensberger ist bei Andersson das Problematische der Literatur und der Sprache nicht, daß sie Welt nur deutend abbilden, es liegt darin, wie sie es tun. Sprache muß zur stimmigen Aussage erst befähigt werden. So wird es denn mehr als eine lediglich zeittypische Wendung, wenn es in dem oben zitierten Aufsatz über die Kritik heißt, Wirklichkeit werde in dem dort geforderten literarischen Abbildverfahren «denkbar», aber auch «veränderbar» und «Püberschreitbar». Sie wird 
das erst in der jeweils experimentell erstellten Antwort auf die bedrängenden Fragen. Hier könnte sich die auf literarisches Sprechen abzielende Erzähltheorie zu einer Theorie der Sprache im allgemeinen erweitern. Arbeiten an der Sprache verändert Denken über Welt. Der Roman richtet das Bewußtsein auf die Bedeutung der schieren Existenz eines Begriffs wie dem des Megatods, er richtet das Augenmerk auf Etymologie.

Es wird verständlich, was Lars Andersson meint, wenn er von Göran Tunström sagt: «Jag tror att Göran Tunström kommer att ha hackat loss en flisa av den gråstarr som ligger mellan språk och värld $»^{18}$. Ohne also das Sprachproblem zu eskamotieren, ohne es schlicht im Stil Steiners lösen zu wollen, wird ein Weg gesucht, über Welt etwas auszusagen, Welt zu erzählen. Anderssons Romane versuchen, unter Rückgriff auf Verfahrensweisen der Moderne - Konstruktion, Experiment - etwas zu leisten, was den heute nicht mehr akzeptierten Theorien der Moderne, die historisch ihren Wert hatten, widerspricht. Hier werden Konsequenzen gezogen aus Denkansätzen, die ihre Überzeugungskraft behalten haben, und es werden Konsequenzen daraus gezogen, daß einige dieser Denkansätze solche Überzeugungskraft nicht mehr besitzen.

Es bliebe zu fragen, wie sich Anderssons Theorie, wie sich seine Praxis zu modernen Autoren anderer Provenienz relationiert, die sich um eine Veränderung der gesprochenen Sprache bemüht haben, die das Verfahren der traditionellen Sprachkritik, zu reglementieren, Denken zu arretieren, beiseite gelegt haben, um eine Befreiung im Sprechen zu erlangen. Hierher gehören Bemühungen einer eher die Semantik als den privaten Geschmack an Sprachlichem bedenkenden Sprachkritik, wie sie wirkungsvoll und überzeugend von Viktor Klemperer vorgeführt wurde. Aber auch die Beatnicks, die Hippies, die Dub-Dichter Jamaicas und alle die, die ihnen u.a. in Skandinavien gefolgt sind, haben - nicht nur intuitiv - an dieser Entwicklung mitgearbeitet. Hier fügt sich Lars Andersson wiederum in diese Entwicklung ein. Hubert Winkels schreibt über Rainald Goetz' Krieg, es sei das «Ideal des Textes: nicht mehr Text zu sein; sondern volle Gegenwart» ${ }^{19}$. Winkels geht auf die Nähe solchen Verfahrens zur Rock-Musik ein, insbesondere zur Heavy Metal-Musik, und tönt seine Erkenntnisse mit Jacques Derrida und denen, die wie Christian Enzensberger argumentieren, $a b$, wenn er als Ergebnis formuliert: «Literatur ist Aufschub, Nachträglichkeit, Sinnzuweisung. Literatur ist Gerede. Die beiden Sphären werden im Namen von Metall und Massaker zusammengeführt. Ein Text soll erzeugt werden, der wie eine Rhythmusmaschine auf Leiber trifft» (159). Goetz entwickle fort, was bei den Beatnicks angefangen habe, weshalb man eine unmittelbare Entwicklungslinie zwischen Rolf Dieter Brinkmann und ihm erkennen könne. Mit Bezug auf Schweden steht er damit in einer Linie mit den literarischen Bemühungen Ulf Lundells, dessen Vinter i paradiset der Erzähler im Prolog zu Anderssons Bikungskupan liest. Auch Lars Andersson hat immer wieder darauf abgehoben, daß Kommunizieren sein Ziel in der communio, daß Sich-Verständigen sein Ziel im Sich-Ver stehen habe, daß aber der Aufschub einerseits, das Gerede mit fremdentsprungener Sinnzuweisung andererseits eine Sperre darstelle, die Kommunikation trotz allen noch so angestrengten Kommunizierens verhindere. In dem von solchem Bewußtsein durchtränkten Versuch, sich zur Gemeinschaft zu verständigen, siegen eher Verzweiflung und Resignation, wie Snöljus zeigt, der Roman, in dem Verständigung nur im sexuellen Vollzug einer erotischen Zweierbeziehung noch gelingt, wie der zur Erzählung übergehende Essay «Överlid» - Om ett efterlämnat manuskript och dess samband med två likfynd på Rävåsmossen ${ }^{20}$ herausstellt, wie es überhaupt die Anstrengungen belegen, Bilder für eine 
sprachlose Kommunikation zu suchen, wie sie bei Bob Dylan etwa gefunden werden, wenn dort der Traum als der Ort erschaffen wird, in dem bei wechselseitiger Teilhabe wortlose Verständigung, Verständigung ohne Aufschub noch Gerede statthabe. Ausgehend von ein und demselben Problem, dem Problem, wie Sprache aussprechen könne, was auszusprechen sie bezweckt, wie ein Bewußtsein davon in Konsequenzen getrieben werden könne, daß hier ein Problem liegt, bemühen sich Autoren wie Lundell und Andersson mit völlig verschiedenen Darbietungsformen und Sprachgesten um Lösungen. Immer wieder stößt man darauf, daß eine Literatur, die verantwortungsvoll verfährt und auf der Höhe unserer Zeit bleiben will, den die Moderne konstituierenden «Zweifel an der Sprache» nicht einfach überspielen kann, daß sie sich diesem Zweifel in immer erneuter Mühe um eine Lösung, und obwohl sie um deren Unzuverlässigkeit weiß, stellen muß.

\section{NOTES}

1. Zit. nach Udo Lindenberg, El Panico oder: Wie werde ich Popstar? Der praktische Ratgeber, München, London, 1988, p. 133.

2. Ib., p. 133 und p. 41.

3. Zit. nach Jan Erik Vold, Poetisk praksis 1975-1990, Oslo, 1990, p. 277.

4. Bo Hakon Jørgensen, «Med sproget oppefra og nede fra til digtet - henimod en overskridelse af modernismen» in NORDICA 8 (1991), pp. 289-299; hier p. 299.

5. Modernism och individualitet. En studie i den litterära modernismens kvalitativa egenart, Lund, 1986, pp. 72 sq.

6. Im folgenden zitiert nach der deutschen Übersetzung: G. St., Von realer Gegenwart. Hat unser Sprechen Inhalt? Mit einem Nachwort von Botho Strauß. Aus dem Englischen von Jörg Trobitius (Edition Akzente), München, Wien, 1990.

7. München, ${ }^{6}-1991\left({ }^{1}-1984\right)$, p. 105.

8. Gunnar Björling, «Min skrift - lyrik?», wiederabgedruckt in G.B., Det stora enklas dag. Dikt och prosa. Urval och inledning av Bo Carpelan, s.l, 1975, pp. 173-192; hier p. 179.

9. «Den moraliska genen», in: L.A., Kattfiske och annat, Stockholm, 1989, pp. 51-59.

10. Abgedruckt in: L.A., Begynnelsebokstäver. Med förord av Staffan Söderblom, Stockholm, 1984, pp. 147-152.

11. Stockholm, 1989.

12. Cf. die Äußerung im ersten Kapitel, in der Gun nar sagt, daß er, hieße er Per Ivar Ödman, ein Gedicht oder ein Drama über Artedi schriebe. Cf. dazu ferner die Entwürfe über Artedi und Majorana im zweiten Teil.

13. Anders als etwa die Prosamythe, die Ödman gegen Ende des ersten Teils des Romans schreibt (99-103), oder als die Strophe (181), mit der Ödman auf eine Strophe eines Gedichts von Moses ibn Ezra (112) reagiert.

14. Im Zusammenhang einer Autorenlesung am 8. Mai 1992 im Nordischen Seminar der Universität Münster bestätigte Lars Andersson meine Vermutung, die hebräischen Buchstaben über den Kapiteln ergäben ein Zitat aus dem Alten Testament, und gab mir freundlicherweise den Hinweis, es handle sich um ein Zitat «aus dem Buch Jesaja». 
15. In seiner Philosophie der Kunst (Hanau, 1909), geht er davon aus, daß Kunst «qualitative Verwandtschaften und Gegensätze» zwischen «verschiedenen Gegenstandsimpressionen» (114) herstelle.

16. Ch.E., Literatur und Interesse. Eine politische Ästhetik mit zwei Beispielen aus der englischen Literatur, Bd. 1: Theorie, München, Wien, 1977, p. 84; cf. auch p. 147 sq.: «die Kunst lügt sobald sie etwas sagt und muß lügen - oder aufhören, ästhetische Struktur und damit Kunst zu sein».

17. Grundbegriffe der Ästhetik (edition suhrkamp, NF 160), Frankfurt/Main, 1938, p. 182.

18. Wie Anm. 10, p. 162.

19. Einschnitte. Zur Literatur der 80er Jahre. Erweiterte und bearbeitete Ausgabe (suhrkamp taschenbuch, 1804), Frankfurt/Main, 1991, p. 259.

20. Wie Anm. 10, pp. 226-250.

\section{RÉSUMÉS}

Die Kritik an der Literatur der Moderne wendete sich gegen deren Kompliziertheit, als die der experimentelle Charakter dieser Literatur empfunden wurde. Dieser experimentelle Charakter kann als Resultat der die Moderne fundierenden Sprachauffassung gedeutet werden. Da Sprachtheorie Teil einer umfassenden Weltdeutung ist, wird die Kritik an der Moderne dort, wo sie mehr ist als emotionale Abwehr des Schwierigen, eine Kritik der philosophischen Grundlagen der gesamten Epoche. George Steiners vor wenigen Jahren vorgelegte größere Auseinandersetzung, die sich zu einer Art Abrechung entwickelte, hat das Sprachproblem in den Mittelpunkt gerückt und die Moderne als «Epoche nach dem Wort », als Epoche des «Epilogs » interpretiert. Seine Auseinandersetzung, der Botho Strauß ein die Kritik noch rigidisierendes Nachwort zugefügt hat, ist in den Konsequenzen antidemokratisch, irrational und biologistisch. Im Werk des schwedischen Autors Lars Andersson findet sich eine Kritik an bestimmten Theorien und Theoremen der Moderne, die der Steiners verwandt ist. Andersson zieht aber andere Konsequenzen aus seiner Problematisierung. Sein Werk, Essayistik wie Epik, stellt sich als eine Fortführung der Moderne im Bewußtsein der Kritik an ihr dar und verdeutlicht, was an der Moderne als unhintergehbar zu betrachten ist und was an der Moderne durch neue Denkansätze überholt ist.

La critique de la littérature moderniste a surtout souligné son caractère compliqué dû au côté expérimental de cette littérature. Cet aspect expérimental peut être interprété comme étant le résultat de la réflexion moderniste sur le langage. Comme la théorie du langage représente une partie intégrante de l'interprétation du monde, la critique du modernisme devient donc, là où elle est davantage qu'un simple refus émotionnel de ce qui est difficile, une critique de l'ensemble des fondements philosophiques d'une époque. Le large Essai que George Steiner a publié il y a quelques années, et qui s'est révélé être une sorte de règlement de compte, a placé le problème du langage au centre, interprétant ainsi le modernisme en tant qu' « époque au-delà du mot", en tant qu'époque de "l'épilogue ». Son analyse, à laquelle Botho Strauss a ajouté une postface amplifiant encore l'aspect rigide de la critique, aboutit à des affirmations du caractère antidémocratique, irrationnel et biologisant.

Dans l'œuvre de l'écrivain suédois Lars Andersson on peut trouver une critique de certaines théories et de certains théorèmes du modernisme qui s'apparente à celle de Steiner. Mais Andersson tire d'autres conclusions de ses réflexions théoriques. Son œuvre - ses essais aussi 
bien que ses écrits épiques - se présente comme une continuation du modernisme à la lumière de la critique qu'on a pu en faire, et montre clairement à la fois ce que le modernisme a finalement d'incontournable et ce que de nouvelles réflexions en ont révélé comme étant caduque.

\section{AUTEUR}

UWE EBEL

Westfälische Wilhelms-Universität Münster 